\title{
Crystal structure of the Fab fragment of burosumab, the first-in-class anti- FGF23 medicine for treating X-linked hypophosphatemia and tumor-induced osteomalacia
}

\author{
Ui Beom Park, Hyun Tae Lee, Yujin Kim, Tae Jun Jeong, Nahyun Gu and Yong-Seok Heo* \\ Department of Chemistry, Konkuk University, Seoul 05029, Republic of Korea \\ *Correspondence: ysheo@konkuk.ac.kr
}

Burosumab is a first-in-class therapy approved to treat X-linked hypophosphatemia and tumor-induced osteomalacia, a condition associated with excessive fibroblast growth factor 23 (FGF23) production. This antibody drug directly targets the excessive FGF23 in patients and inhibits its regulation of phosphate homeostasis and vitamin D metabolism. Here, the Fab fragment of burosumab was expressed, purified, and crystallized. The crystals belonged to the space group $P 2_{1}$, with unit cell parameters $a=49.51, b=66.22, c=62.05 \AA$, and $\beta=105.99^{\circ}$. An asymmetric unit of the crystal contains one Fab fragment with a Matthews coefficient of $1.93 \AA^{3} \mathrm{Da}^{-1}$. The structure was determined at a resolution of $1.45 \AA$, with $R_{\text {work }} / R_{\text {free }}=0.169 / 0.196$. The complementarity determining regions of burosumab construct a negatively charged patch, a putative hot spot for FGF23 binding. This high-resolution structure could be used for predicting the binding mode between burosumab and FGF23.

\section{INTRODUCTION}

Fibroblast growth factors mediate signal transduction involved in autocrine, paracrine, or hormonal activities. Human fibroblast growth factor 23 (FGF23) is a 32 kDa glycoprotein mainly produced in bone and plays an important role as a hormonal regulator of phosphate homeostasis (Saito and Fukumoto, 2009). All endocrine FGFs, including FGF19, FGF21, and FGF23, require co-receptor Klotho ( $\alpha$ - or $\beta$-Klotho) for effective binding to the FGF receptors. The co-receptor $\alpha$-Klotho facilitates the binding of FGF23 to FGFR1c, which is the most critical receptor for FGF23 signaling under physiological conditions. Excessive FGF23 reduces serum levels of phosphorus by regulating phosphate excretion and vitamin D activation in the kidney. For a long time, blocking the excessive FGF23 has been recognized as a promising therapeutic strategy to treat hypophosphatemic rickets (ADHR Consortium, 2000).

In 2018, the U.S. Food and Drug Administration (FDA) approved the first-in-class anti-FGF23 drug, burosumab $\left(\right.$ Crysvita $\left.^{\circledR}\right)$, for treating X-linked hypophosphatemia (XLH), which causes impaired bone growth and development in children (Lamb, 2018). In 2020, the FDA expanded the indications of this drug to include FGF23-related hypophosphatemia in tumorinduced osteomalacia (Whyte, 2021). Burosumab is a fully human monoclonal $\lg 1_{1} \kappa$ antibody targeting FGF23. By directly neutralizing excess activities of FGF23 in patients, burosumab restores phosphate reabsorption by the kidney and increases the activation of vitamin $\mathrm{D}$, which increases gastrointestinal phosphate absorption. Thus, hypophosphatemia and the risk of bone diseases by bone mineralization defects can be significantly reduced.

The structures of human FGF23 and its ternary complex with FGFR1c and $\alpha$-Klotho have established the molecular basis of the interaction of FGF23 with its receptors, thereby enhancing the understanding of the detailed mechanism of FGF23 signaling underlying phosphate homeostasis (Goetz et al., 2007; Chen et al., 2018).

Despite the clinical success of burosumab, no structural information for the antibody and its interaction with FGF23 has been reported to date. To elucidate the mechanism of action and epitope of burosumab, we expressed, purified, and crystallized the Fab fragments of burosumab. Besides, we determined its crystal structure at the resolution of $1.45 \AA$. The detailed understanding of the mechanism of action of burosumab from further structural studies can facilitate the design of improved biologics against FGF23.

\section{RESULTS AND DISCUSSION}

The Fab fragment of burosumab was overexpressed in the periplasmic region of E.coli and purified to homogeneity by the Ni-affinity and size exclusion chromatography (Figure 1A). 
The crystals of the burosumab Fab fragments were generated by a hanging-drop vapor diffusion method with a well solution containing $0.03 \mathrm{M} \mathrm{NaF}, 0.03 \mathrm{M} \mathrm{NaBr}, 0.03 \mathrm{M} \mathrm{Nal}, 20 \%(\mathrm{v} / \mathrm{v})$ PEG500 MME, 10\% (w/v) PEG20000, 0.1 M Sodium HEPES, pH 7.5 at $4{ }^{\circ} \mathrm{C}$ (Figure 1B). X-ray diffraction data were collected to $1.45 \AA$ resolution and the crystals belonged to space group P21, with unit cell parameters $a=49.51, b=66.22, c=62.05 \AA$, and $\beta=105.99^{\circ}$ (Table 1). The Matthews coefficient was calculated to be $1.93 \AA^{3} \mathrm{Da}^{-1}$ with a solvent content of $36.36 \%$ (Matthews, 1968), assuming one Fab molecule in an asymmetric unit. After phasing by molecular replacement, the crystal structure was refined to a resolution of $1.45 \AA$, with $R_{\text {work }} / R_{\text {free }}=0.169 / 0.196$. The asymmetric unit of the crystal contained one molecule of the burosumab Fab with clear electron density on all residues except the residues 132-137 of the heavy chain (Figure 2). The six complementarity determining regions (CDRs) of burosumab would contain the putative paratope for FGF23 binding. Notably, a negatively charged patch is formed by the residues of the CDR regions, including D99, D102, D105 within HCDR3, and D50 of LCDR2, implying its major contribution to FGF23 binding by electrostatic interactions (Figure 3). The high-resolution of the structure would be helpful for predicting the binding mode between burosumab and FGF23. Interestingly, inspection of the surface of FGF23 reveals a highly positively charged spot, which is the heparin-binding site (Figure 4). To obtain further insights on the antigen-antibody interaction, a molecular docking between FGF23 and burosumab was tried using the HADDOCK server with restraints that the acidic and basic residues on the charged spots of each protein participate in interactions (van Zundert et al., 2016). However, the docking result was not reliable, probably due to the conformational flexibility of FGF23.

Further studies to determine the structure of burosumab in complex with FGF23 would elucidate the precise epitope and mechanism of action underlying the blockade of the interaction of FGF23 with FGFR1c or $\alpha$-Klotho. In addition, the structural study can also provide helpful information for the design of improved anti-FGF23 biologics for treating $\mathrm{X}$-linked hypophosphatemia and tumor-induced osteomalacia.
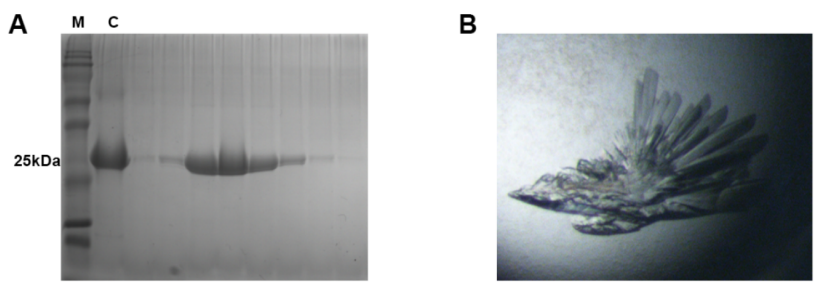

FIGURE 1 I Purification and crystallization of the burosumab Fab. (A) Reducing SDS-PAGE analysis of the fractions of the burosumab Fab after size exclusion chromatography. Lane $\mathrm{M}$ and $\mathrm{C}$ contain molecularweight marker and the sample before SEC, respectively. Due to the similar molecular weight, the heavy and light chains are shown as a single band. (B) Crystals of the burosumab Fab grown in $0.03 \mathrm{M} \mathrm{NaF}, 0.03$ M NaBr, 0.03 M Nal, 20\% PEG500 MME, 10\% PEG20000 0.1 M Sodium HEPES, $\mathrm{pH} 7.5$ at $4^{\circ} \mathrm{C}$.

\section{METHODS}

Expression and purification of the burosumab Fab

The gene encoding the burosumab Fab was synthesized after codon-optimization for $E$. coli expression. The synthesized gene was cloned into a modified pBAD vector using Nhel and Apal restriction sites (Table 2). The construct contains the heavy and light chain with the STII signal sequence in each chain for periplasmic secretion and a $6 \mathrm{xHis}$-tag at the C-terminus of the heavy chain (Lee et al., 2016). The plasmid pBAD-burosumab Fab was transformed into E. coli Top10F (Invitrogen). The cells were grown at $37^{\circ} \mathrm{C}$ in LB medium supplemented with $50 \mu \mathrm{g}$ $\mathrm{ml}^{-1}$ ampicillin. When the OD600 of the culture reached 1.2, the protein expression was induced with $0.2 \%$ arabinose, and cells were further grown at $25^{\circ} \mathrm{C}$ for $15 \mathrm{~h}$. The cells were harvested by centrifugation, re-suspended in lysis buffer $(20 \mathrm{mM}$ Tris, $\mathrm{pH}$ 8.0, $200 \mathrm{mM} \mathrm{NaCl}$ ), and lysed by sonication on ice. After removing cell debris by centrifugation, the burosumab Fab in

TABLE 1 I Data collection and refinement statistics

\begin{tabular}{|c|c|}
\hline Data Collection & \\
\hline X-ray source & PLS 5C \\
\hline Wavelength $(\AA)$ & 1.0000 \\
\hline Space group & $P 2_{1}$ \\
\hline$a, b, c(\AA)$ & $49.51,66.22,62.05$ \\
\hline$\alpha, \beta, \gamma\left(^{\circ}\right)$ & $90,105.99,90$ \\
\hline Resolution $(\AA)$ & $1.45(1.49-1.45)^{\star}$ \\
\hline$R_{\text {sym }}(\%)$ & $10.1(49.5)$ \\
\hline$|/ \sigma|$ & $6.31(1.81)$ \\
\hline Completeness (\%) & 99.7 (85.3) \\
\hline Redundancy & $3.4(2.5)$ \\
\hline $\mathrm{CC}_{1 / 2}$ & $0.985(0.845)$ \\
\hline \multicolumn{2}{|l|}{ Refinement } \\
\hline Resolution $(\AA)$ & 1.45 \\
\hline No. reflections & 68056 \\
\hline$R_{\text {work }} / R_{\text {free }}(\%)$ & 16.9/19.6 \\
\hline \multicolumn{2}{|l|}{ No. atoms } \\
\hline Protein & 3211 \\
\hline Water & 673 \\
\hline Average B-factor $\left(\AA^{2}\right)$ & 22.7 \\
\hline Protein & 21.4 \\
\hline Water & 26.9 \\
\hline \multicolumn{2}{|l|}{ R.m.s. deviation } \\
\hline Bond lengths $(\AA)$ & 0.007 \\
\hline Bond angles $\left({ }^{\circ}\right)$ & 1.239 \\
\hline \multicolumn{2}{|l|}{ Ramachandran } \\
\hline Favored (\%) & 97.61 \\
\hline Allowed (\%) & 2.39 \\
\hline Outlier (\%) & 0.00 \\
\hline PDB code & 7VEN \\
\hline
\end{tabular}

*Values in parentheses are for the outer resolution shell. 
the supernatant was purified using HisTrap HP column (Cytiva) and HiLoad 26/60 Superdex 200 pg column (Cytiva). The elution profile of the protein showed a single major peak in the size exclusion chromatography, and the protein quality was evaluated by SDS-PAGE.

Crystallization, data collection, and structure determination For crystallization, the purified burosumab Fab was concentrated to $20 \mathrm{mg} \mathrm{ml}^{-1}$ in $20 \mathrm{mM}$ Tris, $\mathrm{pH} 8.0$, and $200 \mathrm{mM} \mathrm{NaCl}$. The crystallization was carried out by a vapor-diffusion method with $300 \mu \mathrm{l}$ of the reservoir solution and the hanging drop of $0.8 \mu \mathrm{l}$ of protein solution mixed with $0.8 \mu \mathrm{l}$ of the reservoir solution at $4^{\circ} \mathrm{C}$. Clusters of crystals were grown with a reservoir solution containing $0.03 \mathrm{M} \mathrm{NaF}, 0.03 \mathrm{M} \mathrm{NaBr}, 0.03 \mathrm{M} \mathrm{Nal}, 20 \%$ (v/v) PEG500 MME, 10\% (w/v) PEG20000, 0.1 M Sodium HEPES, $\mathrm{pH} 7.5$ within a week. For cryoprotection, a cluster of crystals was broken with a needle and flash-frozen in liquid nitrogen.

\section{A}
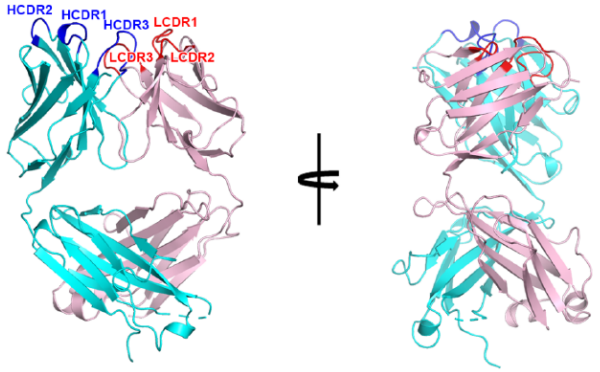

B

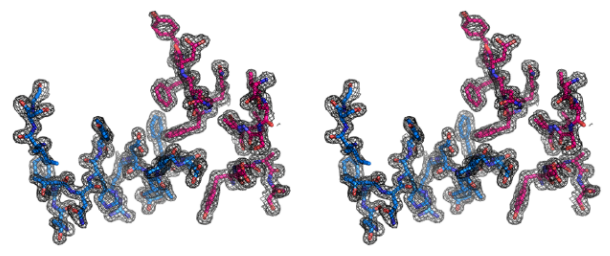

FIGURE 2 I Structure of the Fab fragment of burosumab. (A) Overall structure of the burosumab Fab. The heavy and light chains are colored cyan and pink, respectively. The CDR regions of the heavy and light chains (HCDR1-3 and LCDR1-3) are colored blue and red, respectively. (B) Stereoscopic view of the 2 fofc map ( $1.5 \sigma$ contour level) calculated at 1.45 Å resolution on the CDR regions.

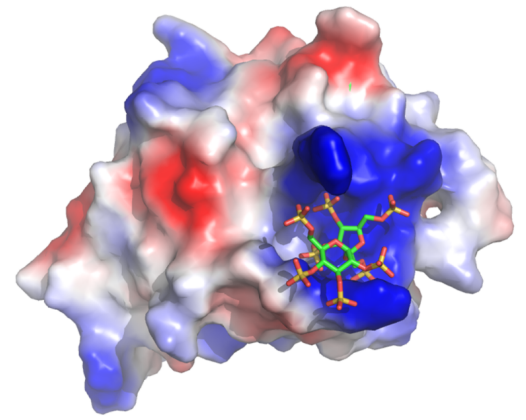

FIGURE 4 I Positively charged spot on the surface of FGF23. Surface representation of the FGF23 with electrostatic potential generated by PyMOL shows a highly positively charged spot on the heparin-binding site (PDB entry 2p93). The bound heparin analogue is represented as a stick model.

TABLE 2 I Macromolecule production

\begin{tabular}{|c|c|}
\hline Protein & Burosumab Fab \\
\hline $\begin{array}{l}\text { Source } \\
\text { organism }\end{array}$ & Burosumab \\
\hline $\begin{array}{l}\text { DNA } \\
\text { source }\end{array}$ & Gene synthesis (Bioneer, Inc) \\
\hline $\begin{array}{l}\text { Restriction } \\
\text { enzyme }\end{array}$ & Nhel and Apal \\
\hline $\begin{array}{l}\text { Cloning } \\
\text { vector }\end{array}$ & pBAD \\
\hline $\begin{array}{l}\text { Expression } \\
\text { vector }\end{array}$ & $\mathrm{pBAD}$ \\
\hline $\begin{array}{l}\text { Expression } \\
\text { host }\end{array}$ & E. coli Top10F \\
\hline $\begin{array}{l}\text { Complete } \\
\text { amino-acid } \\
\text { sequence } \\
\text { of the } \\
\text { construct } \\
\text { produced }\end{array}$ & $\begin{array}{l}<\text { Heavy chain> } \\
\text { QVQLVQSGAEVKKPGASVKVSCKASGYTFTNHYMHWVRQA } \\
\text { PGQGLEWMGIINPISGSTSNAQKFQGRVTMTRDTSTSTVY } \\
\text { MELSSLRSEDTAVYYCARDIVDAFDFWGQGTMVTVSSAST } \\
\text { KGPSVFPLAPSSKSTSGGTALGCLVKDYFPEPVTVSWNS } \\
\text { GALTSGVHTFPAVLQSSGLYSLSSVTVPSSLGTQTYIC } \\
\text { NVNHKPSNTKVDKKVEPKSCDKTHHHHHH } \\
\text { <Light Chain> } \\
\text { AIQLTQSPSSLSASVGDRVTITCRASQGISSALVWYQQKP } \\
\text { GKAPKLLIYDASSLESGVPRFSGSGSGTDFTLTISSLQP } \\
\text { EDFATYYCQQFNDYFTFGPGTKVDIKRTVAAPSVFIFPPS } \\
\text { DEQLKSGTASVVLLNNFYPREAKVQWKVDNALQSGNSQE } \\
\text { SVTEQDSKDSTYSLSSTLTLSKADYEKHKVYACEVTHQGL } \\
\text { SSPVTKSFNRGEC }\end{array}$ \\
\hline
\end{tabular}

A

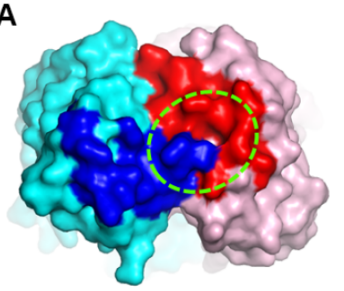

B

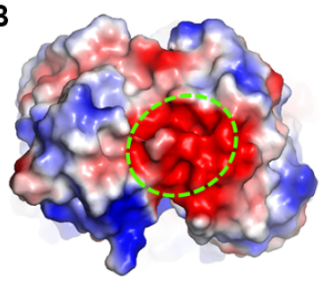

C

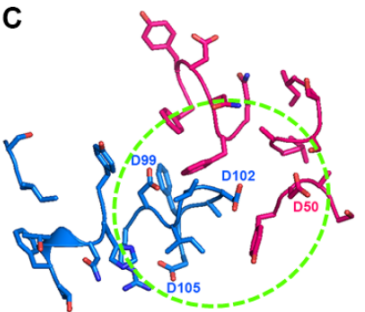

FIGURE 3 I Negatively charged patch on the putative paratope of burosumab. (A) Surface representation of the burosumab Fab. The heavy and light chains are colored cyan and pink, respectively. The CDR regions of the heavy and light chains are colored blue and red, respectively. (B) Electrostatic potential generated by PyMOL on the burosumab surface. (C) The residues within the CDR regions. The acidic residues comprising the negatively charged patch are labeled. The green ellipses indicate the putative region of the negatively charged patch for FGF23 binding. 
X-ray diffraction data for a single crystal were collected at 100 $\mathrm{K}$ on beamline $5 \mathrm{C}$ of the Pohang Light Source (PLS-II) (Park et al., 2017) to a resolution of $1.45 \AA$, integrated and scaled using XDS (Kabsch, 2010). The structure was solved by molecular replacement using Phaser software with the structure of the pembrolizumab Fab (PDB code 5ggs, chains A and B) as a search model (McCoy et al., 2007). The variable and constant regions of the search model were separated during molecular replacement due to the elbow flexibility within the Fab moiety. Model building and structure refinement were carried out using PHENIX and COOT software (Emsley and Cowtan, 2004; Adams et al., 2010). Statistics for data collection and refinement are presented in Table 1. All structural figures were generated using PyMol (Schrödinger, Inc.). The atomic coordinates and structure factors for the crystal structure of the burosumab Fab have been deposited in Protein Data Bank (http://www.rcsb.org), under the entry 7 VEN.

\section{ACKNOWLEDGEMENTS}

We are grateful to the staff of beamline $5 \mathrm{C}$ at Pohang Accelerator Laboratory for help with the X-ray diffraction experiments. This work was supported by a grant from the National Research Foundation of Korea (NRF-2020R1A2C2008865). This paper was written as part of Konkuk University's research support program for its faculty on sabbatical leave in 2020 .

\section{CONFLICT OF INTEREST}

The authors declare no conflict of interest.

\section{AUTHOR INFORMATION}

U.B.P., H.T.L., Y.K., T.J.J., and N.G. performed the experiments. Y.-S.H. wrote the paper. All authors discussed the results and commented on the manuscript.

Original Submission: Sep 10, 2021

Revised Version Received: Sep 19, 2021

Accepted: Sep 27, 2021

\section{REFERENCES}

Adams, P.D., Afonine, P.V., Bunkóczi, G., Chen, V.B., Davis, I.W., Echols, N., Headd, J.J., Hung, L.W., Kapral, G.J., Grosse-Kunstleve, R.W., McCoy, A.J., Moriarty, N.W., Oeffner, R., Read, R.J., Richardson, D.C., et al. (2010). PHENIX: a comprehensive Python-based system for macromolecular structure solution. Acta Crystallogr D Biol Crystallogr 66, 213-221.

ADHR Consortium. (2000). Autosomal dominant hypophosphataemic rickets is associated with mutations in FGF23. Nat Genet 26, 345-348.

Chen, G., Liu, Y., Goetz, R., Fu, L., Jayaraman, S., Hu, M.C., Moe, O.W., Liang, G., Li, X., and Mohammadi, M. (2018). $\alpha$-Klotho is a non-enzymatic molecular scaffold for FGF23 hormone signalling. Nature 553, 461-466.

Emsley, P., and Cowtan, K. (2004). Coot: model-building tools for molecular graphics. Acta Crystallogr D Biol Crystallogr 60, 2126-2132.

Goetz, R., Beenken, A., Ibrahimi, O.A., Kalinina, J., Olsen, S.K., Eliseenkova, A.V., Xu, C., Neubert, T.A., Zhang, F., Linhardt, R.J., Yu, X., White, K.E., Inagaki, T., Kliewer, S.A., Yamamoto, M., et al. (2007). Molecular insights into the klotho-dependent, endocrine mode of action of fibroblast growth factor 19 subfamily members. Mol Cell Biol 27, 34173428.

Kabsch, W. (2010). XDS. Acta Crystallogr D Biol Crystallogr 66, 125-132. Lamb, Y.N. (2018). Burosumab: first global approval. Drugs 78, 707-714.

Lee, J.Y., Lee, H.T., Shin, W., Chae, J., Choi, J., Kim, S.H., Lim, H., Won Heo, T., Park, K.Y., Lee, Y.J., Ryu, S.E., Son, J.Y., Lee, J.U., and Heo, Y.S. (2016). Structural basis of checkpoint blockade by monoclonal antibodies in cancer immunotherapy. Nat Commun 7, 13354.

Matthews, B.W. (1968). Solvent content of protein crystals. J Mol Biol 33, 491-497.

McCoy, A.J., Grosse-Kunstleve, R.W., Adams, P.D., Winn, M.D., Storoni, L.C., and Read, R.J. (2007). Phaser crystallographic software. J Appl Crystallogr 40, 658-674.

Park, S.Y., Ha, S.C., and Kim, Y.G. (2017). The protein crystallography beamlines at the Pohang Light Source II. Biodesign 5, 30-34.

Saito, T., and Fukumoto, S. (2009). Fibroblast growth factor 23 (FGF23) and disorders of phosphate metabolism. Int $J$ Pediatr Endocrinol 2009, 496514.

van Zundert, G.C.P., Rodrigues, J., Trellet, M., Schmitz, C., Kastritis, P.L., Karaca, E., Melquiond, A.S.J., van Dijk, M., de Vries, S.J., and Bonvin, A. (2016). The HADDOCK2.2 web server: user-friendly integrative modeling of biomolecular complexes. J Mol Biol 428, 720-725.

Whyte, M.P. (2021). Tumor-induced osteomalacia: treatment progress using Burosumab, an anti-FGF23 monoclonal antibody. J Bone Miner Res 36, 625-626. 